\title{
Adquisición de Competencias Genéricas en alumnos de Grado y Máster a través de asignaturas de Transportes
}

\author{
Belén Muñoz \\ Prof. Asociado, Universidad Politécnica de Madrid (UPM), España \\ Manuel G. Romana \\ Prof. Titular de Universidad, UPM, España \\ Consuelo Durán \\ Licenciada en Geografía e Historia, UPM, España
}

\section{RESUMEN}

El Espacio Europeo de Educación Superior impone la educación por competencias, lo que obliga a las universidades a establecer las competencias profesionales generales, específicas asociadas a materias y genéricas que adquieren los alumnos con la titulación, a través de las asignaturas del plan de estudios, y las actividades desarrolladas en las mismas.

Hay muchas definiciones de competencias genéricas, pero todas ellas coinciden en destacar que la actuación competente de un profesional implica la aplicación de forma conjunta y coordinada de saberes teóricos, procedimientos, pautas, destrezas, actitudes,.... Por cuestiones de programación, cada competencia genérica no se adquirirá con una única asignatura del plan de estudios, sino con varias de ellas, e incluso con formación complementaria al plan de estudios. Todo ello implica cambios en la metodología docente por parte del profesorado. Según las recomendaciones de ANECA, se trata de definir las competencias que deberán adquirir los alumnos al finalizar la titulación, pero la titulación no solo forma profesionales sino también personas. Por ello, las competencias deberán definirse teniendo en cuenta el respeto a los derechos fundamentales y de igualdad de oportunidades entre hombres y mujeres, los principios de igualdad de oportunidades y accesibilidad universal de las personas con discapacidad y los valores propios de una cultura de la paz y de valores democráticos.

En este trabajo se analiza la adquisición de competencias genéricas por parte de los alumnos de Grado y Máster en asignaturas de Transportes en sus diferentes aspectos, planificación, política, explotación, etc., así como a través de las actividades desarrolladas en las mismas. Para ello se parte como ejemplo de las asignaturas de Transportes incluidas en el plan de estudios de Grado en Ingeniería Civil y Territorial y Máster en Ingeniería de Caminos, Canales y Puertos de la ETSI de Caminos, Canales y Puertos de la UPM.

\section{INTRODUCCIÓN}

La educación por competencias es uno de los objetivos prioritarios de la Universidad Politécnica de Madrid y condición necesaria para la adaptación al Espacio Europeo de Educación Superior (EEES). La educación por competencias supone un cambio importante 
en la actividad docente, y en concreto, la formación y evaluación de las competencias genéricas sigue siendo uno de los aspectos más complicados de abordar por el profesorado, (Muñoz et al., 2015).

Hay muchas definiciones de competencias, las cuales se pueden resumir en que una competencia es un conjunto de conocimientos, actitudes y habilidades que puede ser evaluables y cuyo conjunto permite un desempeño eficaz en un desarrollo profesional. En este sentido, Agencia Nacional de Evaluación de la Calidad y Acreditación, ANECA, indica que por competencia se entiende "el conjunto de conocimientos, habilidades, actitudes que se adquieren o desarrollan mediante experiencias formativas coordinadas, las cuales tienen el propósito de lograr conocimientos funcionales que den respuesta de modo eficiente a una tarea o problema de la vida cotidiana y profesional que requiera un proceso de enseñanza y aprendizaje", (ANECA, 2012). Se puede distinguir dos tipos principales de competencias: específicas asociadas a material y genéricas o transversales. Cada competencia genérica no se adquirirá con una única asignatura del plan de estudios, sino con varias de ellas e incluso con formación complementaria al plan de estudios y además implica cambios en la metodología docente por parte del profesorado.

Desde la implantación del EEES en el sistema universitario español, ha habido una gran proliferación de títulos de Grado y Máster en todo el territorio nacional, este hecho también se ha producido en el ámbito de la Ingeniería Civil, donde se desarrolla la especialidad de Transportes.

Para la determinación y revisión de las competencias de una titulación es muy recomendable que las comisiones responsables del diseño del título, consulten de manera sistemática aquellos colectivos o entidades no universitarias que tengan relación con la titulación (colegios o asociaciones profesionales, empresas de referencia en el sector...), de manera que el perfil del título se ajuste a las demandas sociales y laborales.

Por otra parte, toda competencia genérica que forme parte del plan de estudios debe ser evaluable, para que el alumno pueda acreditar su adquisición con el fin de obtener el título. Es necesario acreditar que el alumno ha adquirido el nivel de dominio exigido para el título concreto y para cada competencia, para ello hay que determinar de forma clara las competencias que se adquieren en cada asignatura del plan de estudios, con qué actividad docente y con qué nivel de dominio. Esto que puede resultar obvio, sin embargo, es un proceso complejo, laborioso y que requiere la implicación de todo personal universitario.

\section{MAPA COMPETENCIAL}

Los requisitos para la verificación de los títulos universitarios oficiales que habilitan para el ejercicio de las profesiones reguladas de Ingeniero Técnico de Obras Públicas e Ingeniero de Caminos, Canales y Puertos están recogidos en las Órdenes Ministeriales CIN/307/2009 
y CIN/309/2009, de 9 de febrero, respectivamente, (Ministerio de Ciencia e Innovación, 2009a; Ministerio de Ciencia e Innovación, 2009b). Estas disposiciones detallan las competencias a adquirir por los alumnos y los créditos ECTS que deben cursar en cada módulo. De igual forma, los planes de estudios deben garantizar la adquisición de las competencias definidas en el Real Decreto 1393/2007, de 29 de octubre, por el que se establece la ordenación de las enseñanzas universitarias oficiales, (Ministerio de Educación y Ciencia, 2007), y las establecidas por la normativa interna de cada Universidad, normalmente competencias genéricas o transversales, (Universidad Politécnica de Madrid, 2008).

Por otra parte, la ETSI de Caminos, Canales y Puertos bajo el amparo de una estrategia global de la UPM de acreditación de los títulos de Ingeniería por parte de la agencia americana para la acreditación de títulos de ciencia, tecnología e ingeniería, ABET, inició el proceso de acreditación de sus títulos en el pasado curso 2013/2014. Este hecho obligó a realizar el seguimiento y evaluación de una serie de competencias establecidas en la reglamentación de ABET, las cuales pueden ser específicas de materias, asociadas a materias pero transversales a varias asignaturas o materias y competencias genéricas, (ABET, 2014).

Para dar cumplimiento a todos los requerimientos anteriores y aportar un poco de claridad y sencillez dentro de este maremágnum de competencias, la Junta de Escuela de la Escuela de Caminos, Canales y Puertos aprobó el mapa competencial del Grado en Ingeniería Civil y Territorial y el Máster de Ingeniería de Caminos, Canales y Puertos, los cuales han quedado agrupado en las competencias definidas por ABET. De manera que se ha establecido una correspondencia entre competencias profesionales y específicas de las materias y las competencias genéricas definidas en las memorias de verificación con las competencias definidas por ABET, junto con cuatro competencias genéricas, de forma que sea más sencilla la evaluación y acreditación de competencias genéricas.

De esta forma, las competencias genéricas que los alumnos de ambas de titulaciones deben adquirir, son las siguientes: 


\begin{tabular}{|l|l|l|}
\hline \multirow{4}{*}{ ABET } & F & Conocimiento de la responsabilidad ética y profesional \\
\cline { 2 - 3 } & G & Capacidad para comunicar eficazmente \\
\cline { 2 - 3 } & I & $\begin{array}{l}\text { Reconocimiento de la necesidad de aprendizaje a lo largo de la } \\
\text { vida }\end{array}$ \\
\cline { 2 - 3 } & J & Conocimiento de las cuestiones actuales y contemporáneas \\
\hline \multirow{2}{*}{$\begin{array}{l}\text { ETSI } \\
\text { Caminos }\end{array}$} & L & Conocimiento de idiomas \\
\cline { 2 - 3 } & M & Conciencia del cuidado por el Medio Ambiente \\
\cline { 2 - 3 } & $\mathrm{N}$ & Transferencia tecnológica y cooperación \\
\cline { 2 - 3 } & O & Creatividad \\
\hline
\end{tabular}

Tabla 1: Competencias genéricas de la titulación de Grado en Ingeniería Civil y Territorial y del Máster en Ingeniería de Caminos, Canales y Puertos, Universidad Politécnica de Madrid.

\section{ASIGNATURAS DE TRANSPORTES EN EL PLAN DE ESTUDIOS.}

Según se establece en la memoria de verificación del título de Grado en Ingeniería Civil y Territorial de la Universidad Politécnica de Madrid aprobada por ANECA, (ETSI DE CAMINOS, CANALES Y PUERTOS, 2009), las asignaturas de Transportes se encuentran distribuidas en el Plan de Estudios de la siguiente forma:

\begin{tabular}{|c|c|c|}
\hline \multirow{3}{*}{$\begin{array}{l}\text { Mención de } \\
\text { Construcciones } \\
\text { Civiles }\end{array}$} & \multirow{2}{*}{$\begin{array}{l}\text { Séptimo semestre } \\
\left(4^{\circ} \text { curso }\right)\end{array}$} & $\begin{array}{l}\text { Caminos para la especialidad de Construcciones } \\
\text { Civiles }\end{array}$ \\
\hline & & Transportes \\
\hline & $\begin{array}{l}\text { Octavo semestre } \\
\left(4^{\circ} \text { curso }\right)\end{array}$ & $\begin{array}{l}\text { Ferrocarriles para la especialidad de } \\
\text { Construcciones Civiles }\end{array}$ \\
\hline $\begin{array}{l}\text { Mención de } \\
\text { Hidrología }\end{array}$ & $\begin{array}{l}\text { Séptimo semestre } \\
\left(4^{\circ} \text { curso }\right)\end{array}$ & Caminos para la especialidad de Hidrología \\
\hline \multirow{7}{*}{$\begin{array}{lr}\text { Mención de } \\
\text { Transportes } \\
\text { Servicios } & \\
\text { Urbanos } & \end{array}$} & \multirow{4}{*}{$\begin{array}{l}\text { Séptimo semestre } \\
\left(4^{\circ} \text { curso }\right)\end{array}$} & $\begin{array}{l}\text { Caminos para la especialidad de Transportes y } \\
\text { Servicios Urbanos }\end{array}$ \\
\hline & & Transportes \\
\hline & & Explotación Portuaria \\
\hline & & Transportes Urbanos \\
\hline & \multirow{3}{*}{$\begin{array}{l}\text { Octavo semestre } \\
\left(4^{\circ} \text { curso }\right)\end{array}$} & Tráfico y Seguridad Viaria \\
\hline & & Ferrocarriles \\
\hline & & Firmes y pavimentos \\
\hline
\end{tabular}

Tabla 2: Asignaturas de Transportes del Plan de Estudios de la titulación de Grado en Ingeniería Civil y Territorial, Universidad Politécnica de Madrid

De igual forma, en la memoria de verificación del título de Máster en Ingeniería de Caminos, Canales y Puertos de la Universidad Politécnica de Madrid aprobada por ANECA, (ETSI DE CAMINOS, CANALES Y PUERTOS, 2013), se establecen las asignaturas de 
Transportes que forman del Plan de Estudios, y se distribuyen de la siguiente forma:

\begin{tabular}{|c|c|c|}
\hline \multirow{3}{*}{$\begin{array}{l}\text { Asignaturas } \\
\text { obligatorias }\end{array}$} & $\begin{array}{l}\text { Primer semestre } \\
\left(1^{\circ} \text { curso }\right)\end{array}$ & Sistemas de Transporte \\
\hline & $\begin{array}{l}\text { Segundo semestre } \\
\left(1^{\mathrm{o}} \text { curso }\right)\end{array}$ & Sistemas Ferroviarios \\
\hline & $\begin{array}{l}\text { Tercer semestre } \\
\left(2^{\circ} \text { curso }\right)\end{array}$ & Planificación y Gestión de Carreteras \\
\hline \multirow{6}{*}{$\begin{array}{l}\text { Mención de } \\
\text { Transportes, } \\
\text { Territorio y } \\
\text { Urbanismo }\end{array}$} & \multirow{2}{*}{$\begin{array}{l}\text { Tercer semestre } \\
\left(2^{\circ} \text { curso }\right)\end{array}$} & Tecnología ferroviaria \\
\hline & & Gestión Portuaria \\
\hline & \multirow{4}{*}{$\begin{array}{l}\text { Cuarto semestre } \\
\left(2^{\circ} \text { curso }\right)\end{array}$} & Modelos de Demanda de Transporte \\
\hline & & Aeropuertos \\
\hline & & Diseño Viario \\
\hline & & Gestión de la Circulación Viaria \\
\hline
\end{tabular}

Tabla 3: Asignaturas de Transportes del Plan de Estudios de la titulación de Máster en Ingeniería de Caminos, Canales y Puertos, Universidad Politécnica de Madrid

\section{MATRIZ COMPETENCIAS-ASIGNATURAS-ACTIVIDADES}

Hay que tener en cuenta que el proceso de adquisición y desarrollo de competencias genéricas por parte de los alumnos es un proceso largo, que habitualmente no puede realizarse con una única asignatura. También hay que recordar en este punto, que en la adquisición de competencias genéricas, el alumno no aprende lo mismo cuando realiza la actividad por primera vez que cuando la realiza en sucesivas ocasiones, en las cuales su nivel de aprendizaje crece pero a un ritmo más lento. Si representáramos este proceso gráficamente obtendríamos una curva similar a una curva tipo Pareto, (Muñoz et al., 2015).

De forma general, en la ETSI de Caminos, Canales y Puertos, se han determinado una serie de actividades que se desarrollan en las diferentes asignaturas, y así como, otras actividades no vinculadas a una asignatura determinada, pero que también permiten al alumno adquirir las competencias genéricas que acredita el título una vez que finaliza sus estudios, como seminarios, participación en asociaciones, talleres, etc. Entre las actividades incluidas en asignaturas, se pueden indicar las siguientes:

- Presentaciones orales

- Análisis de artículos de investigación

- Representación de distintos roles en resolución de casos prácticos del tipo resolución de conflictos y situaciones reales de la vida profesional.

- Realización de trabajos en grupo.

- Resolución de casos prácticos.

- Realización de proyectos. 
- Realización de trabajos individuales.

Centrando la comunicación en las asignaturas de Transportes, que es el objeto de la misma, a continuación se incluyen las actividades desarrolladas en las asignaturas del área de Transportes del plan de estudios, para ello se incluye lo que se conoce como la Matriz de Asignaturas - Actividades - Competencias:

\begin{tabular}{|c|c|c|}
\hline ASIGNATURAS & ACTIVIDADES & COMPETENCIAS \\
\hline $\begin{array}{l}\text { Caminos para la especialidad de } \\
\text { Construcciones Civiles }\end{array}$ & $\begin{array}{l}\text { Resolución en grupo por roles de un } \\
\text { trabajo práctico }\end{array}$ & $\mathrm{D}, \mathrm{G}, \mathrm{I}, \mathrm{M}, \mathrm{O}$ \\
\hline Transportes & $\begin{array}{l}\text { Resolución de ejercicios en grupos de } \\
\text { trabajo }\end{array}$ & $\mathbf{D}, \mathbf{J}$ \\
\hline $\begin{array}{l}\text { Ferrocarriles para la especialidad de } \\
\text { Construcciones Civiles }\end{array}$ & $\begin{array}{l}\text { Resolución de trabajos y exposición oral } \\
\text { en clase }\end{array}$ & $\mathbf{D}, \mathbf{G}, \mathbf{O}$ \\
\hline $\begin{array}{l}\text { Caminos para la especialidad de } \\
\text { Hidrología }\end{array}$ & $\begin{array}{l}\text { Resolución en grupo por roles de un } \\
\text { trabajo práctico }\end{array}$ & D, G, I, M, O \\
\hline $\begin{array}{l}\text { Caminos para la especialidad de } \\
\text { Transportes y Servicios Urbanos }\end{array}$ & $\begin{array}{l}\text { Resolución en grupo por roles de un } \\
\text { trabajo práctico }\end{array}$ & D, G, I, M, O \\
\hline Transportes & $\begin{array}{l}\text { Resolución de ejercicios en grupos de } \\
\text { trabajo }\end{array}$ & $\mathbf{D}, \mathbf{J}$ \\
\hline Explotación Portuaria & $\begin{array}{l}\text { Resolución de casos prácticos en talleres. } \\
\text { Relación de trabajos en grupo }\end{array}$ & $\mathbf{D}, \mathbf{F}$ \\
\hline Transportes Urbanos & $\begin{array}{l}\text { Resolución interactiva de casos prácticos } \\
\text { de forma individual } \\
\text { Resolución en grupo de ejercicios o casos } \\
\text { prácticos }\end{array}$ & $\mathbf{D}, \mathbf{J}, \mathbf{N}$ \\
\hline Tráfico y Seguridad Viaria & $\begin{array}{l}\text { Realización de casos prácticos para } \\
\text { aplicación de los conocimientos } \\
\text { adquiridos a situaciones reales, resolución } \\
\text { de problemas similares a los que se } \\
\text { encontrará en la vida profesiona }\end{array}$ & F, I, J, N \\
\hline $\begin{array}{l}\text { Ferrocarriles para la especialidad de } \\
\text { Transportes y Servicios Urbanos }\end{array}$ & $\begin{array}{l}\text { Resolución de trabajos y exposición oral } \\
\text { en clase }\end{array}$ & $\mathrm{D}, \mathrm{G}, \mathrm{O}$ \\
\hline Firmes y Pavimentos & $\begin{array}{l}\text { Resolución de caso práctico de estudio de } \\
\text { materiales, y realización de documento } \\
\text { impreso con los resultados de ensayos de } \\
\text { laboratorio. } \\
\text { Talleres de oficina técnica en los cuales se } \\
\text { desarrollarán, colectivamente, casos } \\
\text { prácticos o "tareas tipo" propias de la } \\
\text { ingeniería de Firmes y Pavimentos. }\end{array}$ & D, N \\
\hline
\end{tabular}

Tabla 4. Matriz de Asignaturas - Actividades - Competencias, asignaturas de Transportes del título de Grado en Ingeniería Civil y Territorial, UPM.

A la vista de la tabla anterior, se puede determinar el número de asignaturas de Transportes que contribuyen, a través de las actividades desarrolladas, a la adquisición de las competencias genéricas aprobadas por la Junta de Escuela.

\section{COMPETENCIA}

\begin{tabular}{c|c|}
$\mathbf{N}^{\mathbf{0}}$ & $\%$ \\
Asignaturas & Sobre el total \\
\hline
\end{tabular}




\begin{tabular}{|l|c|c|}
\hline D, Capacidad para trabajar en equipos multidisciplinares & 10 & $91 \%$ \\
\hline F, Conocimiento de la responsabilidad ética y profesional & 2 & $18 \%$ \\
\hline $\begin{array}{l}\text { G, Capacidad para comunicar eficazmente } \\
\begin{array}{l}\text { I, Reconocimiento de la necesidad de aprendizaje a lo } \\
\text { largo de la vida }\end{array}\end{array}$ & 5 & $45 \%$ \\
\hline $\begin{array}{l}\text { J, Conocimiento de las cuestiones actuales y } \\
\text { contemporáneas }\end{array}$ & 4 & $36 \%$ \\
\hline $\begin{array}{l}\text { L, Conocimiento de idiomas } \\
\text { M, Conciencia del cuidado por el Medio Ambiente }\end{array}$ & 3 & $0 \%$ \\
\hline N, Transferencia tecnológica y cooperación & 5 & $27 \%$ \\
\hline O, Creatividad & 3 & $27 \%$ \\
\hline
\end{tabular}

Tabla 5. Asignaturas (en número y en porcentaje) que permiten la adquisición de competencias genéricas, título de Grado en Ingeniería Civil y Territorial, UPM.

Ante los resultados anteriores, y para que no se repitan actividades que contribuyen a adquirir la misma competencia genérica en diferentes asignaturas o por el contrario alguna competencia quede sin ser contemplada, sería necesario revisar las actividades de las asignaturas de forma global en toda el área de Transportes. En este sentido, habría que revisar y distribuir las actividades de trabajo en grupo para que no se repitan a lo largo de las diferentes asignaturas, al mismo tiempo que habría reforzar las actividades que contribuyen a la adquisición de la competencia $\mathrm{F}$, e introducir más docencia en inglés porque la competencia L, Conocimiento de idiomas, no está contemplada en ninguna asignatura. 


\begin{tabular}{|c|c|c|}
\hline ASIGNATURAS & ACTIVIDADES & COMPETENCIAS \\
\hline Sistemas de Transporte & $\begin{array}{l}\text { Análisis en grupos de las tecnologías y } \\
\text { soluciones de transporte. } \\
\text { Realización en grupo de trabajos de } \\
\text { búsqueda y análisis de información, } \\
\text { discusión con los otros miembros del } \\
\text { grupo redacción de un documento de } \\
\text { conclusiones, así como presentación y } \\
\text { defensa pública. }\end{array}$ & $\mathrm{D}, \mathbf{F}, \mathbf{G}, \mathbf{J}, \mathbf{N}, \mathbf{O}$ \\
\hline Sistemas Ferroviarios & $\begin{array}{l}\text { Realización de trabajos específicos y } \\
\text { profesionales en grupo }\end{array}$ & $\mathbf{D}$ \\
\hline Planificación y Gestión de Carreteras & $\begin{array}{l}\text { Realización de tres a seis casos prácticos, } \\
\text { cada uno de los cuales será analizado y } \\
\text { resuelto en grupos }\end{array}$ & $\mathrm{D}, \mathbf{F}, \mathbf{N}$ \\
\hline Tecnología ferroviaria & $\begin{array}{l}\text { Realizacion de trabajos individuales. } \\
\text { Realización de casos prácticos en grupo }\end{array}$ & $\mathbf{D}$ \\
\hline Gestión Portuaria & $\begin{array}{l}\text { Realizacion de trabajos individuales. } \\
\text { Realización de casos prácticos en grupo }\end{array}$ & $\mathrm{D}, \mathrm{G}, \mathrm{I}, \mathrm{O}$ \\
\hline Modelos de Demanda de Transporte & $\begin{array}{l}\text { Realización de casos prácticos } \\
\text { individuales ye } n \text { grupo. } \\
\text { Realización de casos prácticos con } \\
\text { herramientas informáticas de modelización } \\
\text { de tráfico. } \\
\text { Realización de trabajos de búsqueda y } \\
\text { análisis de información. }\end{array}$ & D, I, J, L, N \\
\hline Aeropuertos & $\begin{array}{l}\text { Las clases prácticas }(60 \%) \text { se realizan por } \\
\text { grupos de alumnos en talleres para la } \\
\text { resolución de ejercicios prácticos, } \\
\text { similares a los de la vida profesional, que } \\
\text { se expondrán oralmente en clase. }\end{array}$ & D, F, G, J \\
\hline Diseño Viario & $\begin{array}{l}\text { Realizacion de ejercicios prácticos que se } \\
\text { realizan en grupos. } \\
\text { Realización de un trabajo individual que } \\
\text { incluirá una búsqueda bibliográfica y el } \\
\text { análisis de textos técnicos (artículos, } \\
\text { capítulos de libros...), el cual se expondrá } \\
\text { en clase. }\end{array}$ & D, G, J \\
\hline Gestión de la Circulación Viaria & $\begin{array}{l}\text { Realización de forma individual de } \\
\text { ejercicios prácticos, parte de los cuales se } \\
\text { exponen oralmente. } \\
\text { Realización de un trabajo dirigido por el } \\
\text { profesor que verse sobre uno de los } \\
\text { bloques temáticos de la asignatura, similar } \\
\text { a un trabajo profesional. }\end{array}$ & $\mathbf{F}, \mathbf{G}, \mathbf{I}, \mathbf{J}, \mathbf{N}$ \\
\hline
\end{tabular}

Tabla 6. Matriz de Asignaturas - Actividades - Competencias, asignaturas de Transportes del título de Máster en Ingeniería de Caminos, Canales y Puertos, UPM.

De igual forma que en el caso del título de Grado, es conveniente contabilizar el número de asignaturas que contribuyen a la adquisición de las competencias genéricas. 


\begin{tabular}{|l|c|c|}
\hline \multicolumn{1}{|c|}{ COMPETENCIA } & $\begin{array}{c}\mathbf{N}^{\mathbf{0}} \\
\text { Asignaturas }\end{array}$ & $\begin{array}{c}\% \\
\text { Sobre } \\
\text { el total }\end{array}$ \\
\hline D, Capacidad para trabajar en equipos multidisciplinares & 8 & $89 \%$ \\
\hline F, Conocimiento de la responsabilidad ética y profesional & 3 & $33 \%$ \\
\hline G, Capacidad para comunicar eficazmente & 5 & $56 \%$ \\
\hline $\begin{array}{l}\text { I, Reconocimiento de la necesidad de aprendizaje a lo largo de la } \\
\text { vida }\end{array}$ & 2 & $22 \%$ \\
\hline J, Conocimiento de las cuestiones actuales y contemporáneas & 5 & $56 \%$ \\
\hline L, Conocimiento de idiomas & 1 & $11 \%$ \\
\hline M, Conciencia del cuidado por el Medio Ambiente & 0 & $0 \%$ \\
\hline N, Transferencia tecnológica y cooperación & 4 & $44 \%$ \\
\hline O, Creatividad & 2 & $22 \%$ \\
\hline
\end{tabular}

Tabla 7. Asignaturas (en número y en porcentaje) que permiten la adquisición de competencias genéricas, título de Máster en Ingeniería de Caminos, Canales y Puertos, UPM.

Ante estos datos, y para que evitar duplicidades o carencias de actividades que contribuyen a la adquisición de competencias genéricas, es necesario revisar y redistribuir las actividades a desarrollar por parte de los alumnos en las diferentes asignaturas de Transportes del título de Máster. Por este motivo, habría que revisar y distribuir las actividades de trabajo en grupo para que no se repitan a lo largo de las diferentes asignaturas, al igual que ocurre en el título de Grado, y al mismo tiempo que habría reforzar las actividades que contribuyen a la adquisición de la competencia L, aumentando la docencia en inglés, e introducir actividades que contribuyan a la adquisición de la competencia M, Conciencia por el cuidado del Medio Ambiente, no está contemplada en ninguna asignatura.

Estas tablas y matrices de competencias genéricas constituyen una herramienta eficaz para el control, seguimiento y registro de las competencias genéricas.

\section{CONCLUSIONES}

- Las asignaturas de Transportes de ambos títulos, a través de las actividades que se desarrollan bajo su ámbito, permiten que los que estudiantes adquieran prácticamente la totalidad de las competencias genéricas aprobadas por la Junta de Escuela.

- La definición de Matriz de Asignaturas - Actividades - Competencias para todas las asignaturas de Transportes, constituye una herramienta eficaz para el control, seguimiento y registro de las competencias genéricas por parte de Jefatura de Estudios y de la Dirección del Departamento responsable de dichas asignaturas.

- Ante los resultados obtenidos e incluidos en las tablas 5 y 7 , es necesario redefinir y distribuir las actividades que se desarrollan en las diferentes asignaturas del área de 
Transportes. Este ejercicio de revisión y registro debe realizarse de forma anual para tener un correcto seguimiento de la adquisición de competencias genéricas por parte de los alumnos.

\section{REFERENCIAS}

ABET, (2014). Criteria for Accrediting Applied Science Programs, 2014 - 2015. Accreditation Criteria \& Supporting Documents. www.abet.org.

ANECA, (2012). Guía de Apoyo para la elaboración de la memoria para la solicitud de verificación de títulos oficiales (Grado y Máster). Agencia Nacional de Evaluación de la Calidad y Acreditación, ANECA.

ETSI DE CAMINOS, CANALES Y PUERTOS, (2009). Memoria de Verificación del título de Graduado en Ingeniería Civil y Territorial de la Universidad Politécnica de Madrid. Universidad Politécnica de Madrid.

ETSI DE CAMINOS, CANALES Y PUERTOS, (2013). Memoria de Verificación del título de Máster en Ingeniería de Caminos, Canales y Puertos de la Universidad Politécnica de Madrid. Universidad Politécnica de Madrid.

MINISTERIO DE CIENCIA E INNOVACIÓN, (2009). Orden CIN/307/2009, de 9 de febrero, por la que se establecen los requisitos para la verificación de los títulos universitarios oficiales que habiliten para el ejercicio de la profesión de Ingeniero Técnico de Obras Públicas. Gobierno de España.

MINISTERIO DE CIENCIA E INNOVACIÓN, (2009). Orden CIN/309/2009, de 9 de febrero, por la que se establecen los requisitos para la verificación de los títulos universitarios oficiales que habiliten para el ejercicio de la profesión de Ingeniero de Caminos, Canales y Puertos. Gobierno de España.

MINISTERIO DE EDUCACION Y CIENCIA, (2007). Real Decreto 1393/2007, de 29 de octubre, por el que se establece la ordenación de las enseñanzas universitarias oficiales. Gobierno de España.

MUÑOZ, B., ROMANA, M., DURAN, C., (2015). Sistemas de Medición y Centralización de las Competencias Genéricas del Grado de Ingeniería Civil y Máster de Ingeniería de Caminos, 8-10 abril de 2015, comunicación y ponencia. Congreso Internacional Observal 2015, Cualificaciones Profesionales y Acreditación de Competencias, Valladolid, España.

UNIVERSIDAD POLITÉCNICA DE MADRID, (2008). Consejo de Gobierno de la UPM: Requisitos y recomendaciones para la implantación de Planes de Estudio en la Universidad 
Politécnica de Madrid. Universidad Politécnica de Madrid. 